\title{
Expression of matrix metalloproteinase-10 in non-metastatic prostate cancer: Correlation with an imbalance in cell proliferation and apoptosis
}

\author{
SUGURE MARUTA $^{1}$, YASUYOSHI MIYATA ${ }^{1}$, YUJI SAGARA ${ }^{1}$, SHIGERU KANDA ${ }^{2}$, TAKAHISA IWATA ${ }^{1}$, \\ SHIN-ICHI WATANABE ${ }^{1}$, HIDEKI SAKAI ${ }^{1}$, TOMAYOSHI HAYASHI ${ }^{3}$ and HIROSHI KANETAKE ${ }^{1}$ \\ ${ }^{1}$ Department of Urology, Nagasaki University Graduate School of Biomedical Sciences; ${ }^{2}$ Nagasaki Hospital, \\ National Hospital Organization; ${ }^{3}$ Department of Pathology, Nagasaki University Hospital, Nagasaki, Japan
}

Received June 25, 2009; Accepted January 19, 2010

DOI: 10.3892/ol_00000073

\begin{abstract}
Matrix metalloproteinases (MMPs) are associated with cell invasion under various physiological and pathological conditions. Among MMPs, MMP-10 is reported to correlate with a high $\mathrm{pT}$ stage and progression in a variety of cancer types. However, the clinical and pathological significance of MMP-10 in human prostate cancer tissues remains unclear. This study aimed to clarify the role of MMP-10 in non-metastatic prostate cancer. Sixty-three specimens were obtained by radical prostatectomy. MMP-10 expression, $\mathrm{Ki}-67, \mathrm{CD} 34$ and apoptotic cells were examined using an immunohistochemical technique and the terminal deoxynucleotidyl transferase-mediated nick end-labeling method. The proliferation index (PI), apoptotic index (AI), microvessel density (MVD) and cell renewal index $(\mathrm{CRI}=\mathrm{PI} / \mathrm{AI})$ were calculated. The relationship between MMP-10 expression and clinicopathological features, as well as PI, AI, MVD and CRI were investigated. MMP-10 was mainly detected in cancer cell cytoplasm, and the proportion of MMP-10-expressing cancer cells (median 13.8\%) was significantly higher $(\mathrm{P}<0.001)$ than non-tumoral gland cells (2.4\%). Similarly, the proportion of MMP-10-expressing cancer cells was significantly higher $(\mathrm{P}=0.007)$ in stage $\mathrm{pT} 3$ (median 22.3\%) than in pT2 (11.3\%) tumors and was correlated with blood vessel invasion ( $\mathrm{P}=0.025)$. In addition, its expression level correlated significantly with $\mathrm{CRI}(\mathrm{r}=0.34, \mathrm{P}=0.001)$, but not with PI, AI or MVD. Multivariate analysis identified MMP-10 expression to be closely associated with $\mathrm{pT}$ stage (OR 3.76, 95\% CI 1.14-12.34, $\mathrm{P}=0.029$ ). Our results suggest that the overexpression of MMP-10 produces an imbalance in cancer cell proliferation and apoptosis, thereby contributing to cancer cell progression of non-metastatic prostate cancer.
\end{abstract}

Correspondence to: Dr Yasuyoshi Miyata, Department of Urology, Nagasaki University Graduate School of Biomedical Science, 1-7-1 Sakamoto, Nagasaki 852-8501, Japan

E-mail: int.doc.miya@m3.dion.ne.jp

Key words: matrix metalloproteinase-10, proliferation, apoptosis, $\mathrm{T}$ stage, prostate cancer

\section{Introduction}

Prostate cancer is the most common urological malignancy in elderly individuals and is one of the leading causes of male malignancy-related death worldwide. In the normal prostate gland, cell proliferation equilibrates with cell death, in order that disproportionate overgrowth does not occur. On the other hand, many studies have demonstrated that an imbalance in the growth equation is one of the etiological factors of tumor progression. In other words, the up-regulation of cancer cell proliferation and/or deregulation of apoptosis have been reported to play crucial roles in carcinogenesis and malignant activities in various malignancies, including prostate cancer $(1,2)$. In addition, angiogenesis is an important regulator of malignant aggressiveness, while dissemination via blood vessels is an important process for prognosis (3). Consequently, investigations regarding the regulator of these activities are important for determining treatment strategies in patients with prostate cancer.

Matrix metalloproteinases (MMPs) are zinc-dependent proteolytic enzymes capable of cleaving extracellular matrix and basement membrane macromolecules. Since the degradation of such components is an important process in cancer cell invasion, much interest has centered on the relationship between MMPs and tumor progression in various malignancies $(4,5)$. In addition to such invasive activity, certain MMPs exhibit various pathological functions, such as cell proliferation, apoptosis and angiogenesis (4-6). Thus, we hypothesized that a variety of MMPs modulate cancer cell progression and survival in patients with prostate cancer. Although MMP-2 and -9 are the most representative and well-studied members of the MMP family (7), the clinical and pathological significance of other MMPs in patients with prostate cancer is not fully understood.

With regard to the therapeutic as well as the side effects of MMP inhibitors in cancer patients, disappointing results have been shown against expectations, probably due to the broad specificity of these effects (8). However, specific MMP inhibitors were previously developed, and several investigators examined their effects on the prevention and treatment of various malignancies $(9,10)$. Thus, understanding the detailed 
clinical significance and pathological roles of MMPs is important in the determination of treatment strategies for cancer patients. MMP-10 (stromelysin-2) is overexpressed in various cancer cells, and its expression level correlates with malignant aggressiveness, including invasion $(11,12)$. Moreover, several investigators reported that MMP-10 plays a role in cell proliferation, apoptosis and angiogenesis under certain physiological and pathological conditions $(13,14)$. However, to the best of our knowledge, little is known as regards the clinical significance and role of MMP-10 in human prostate cancer tissues.

In prostate cancer, when the cells are localized in the prostate, the majority of patients present few symptoms. Conversely, clinical symptoms that affect quality of life, such as bone pain and urination disorders, are observed in prostate cancer patients with distant metastasis. In addition, most prostate cancer-related deaths are not the result of the primary tumor but the spread of cancer cells into surrounding tissues and distant organs. In general, up-staging of the $\mathrm{T}$ stage precedes the formation of metastatic tumor. Consequently, investigations concerning the mechanism of early stages in cancer cell invasion and tumor growth are important in the planning of effective treatment strategies in patients with prostate cancer.

The present study aimed to clarify the clinical and pathological significance of MMP-10 in human non-metastatic prostate cancer. The relationship between MMP-10 expression and cancer cell proliferation, apoptosis and angiogenesis were also investigated.

\section{Materials and methods}

Patients. We retrospectively evaluated the medical records of prostate cancer patients who underwent radical surgery between March 1994 and November 2007. To evaluate the preoperative status of metastasis, patients underwent ultrasonography, computed tomography (CT) of the abdomen and pelvis, bone scanning and lung X-ray photography. Pathological diagnoses, including Gleason's score (GS) and pT and pN stage, were determined using specimens obtained during surgery, and staging was assessed by the 2002 tumornode-metastasis classification. In addition to the pathological diagnosis, vascular invasion was judged by one pathologist (T.H.). Patients with metastatic cancers were excluded. None of the patients had received neoadjuvant therapy. After excluding the patients to whom the above criteria were applicable, 63 patients were enrolled in the study; there was no patient with stage pT4. In addition, control samples $(n=40)$ of normal prostate tissues obtained by transurethral resection were also examined. For statistical analyses, GS was divided into the groups: low $(\leq 6)$, middle $(7)$ and high $(\geq 8)$. The study protocol complied with the regulations of the Human Ethics Review Committee of Nagasaki University Graduate School of Biomedical Science.

Immunohistochemistry. Sections $(5-\mu \mathrm{m})$ cut from formalin-fixed and paraffin-embedded tissue samples were deparaffinized and rehydrated. Antigen retrieval was performed at $95^{\circ} \mathrm{C}$ for $40 \mathrm{~min}$ (for MMP-10 and CD34) and at $121^{\circ} \mathrm{C}$ for $15 \mathrm{~min}$ (for $\mathrm{Ki}-67$ ) in $0.01 \mathrm{M}$ sodium citrate buffer ( $\mathrm{pH}$ 6.0). Sections were then immersed in $3 \%$ hydrogen peroxide for $30 \mathrm{~min}$. Primary antibodies were obtained from Lab Vision Corporation, Freemont, CA, USA (MMP-10) and Dako Corp., Glostrup, Denmark (Ki-67 and CD34). The sections were then incubated with the primary antibody at $4{ }^{\circ} \mathrm{C}$ overnight. Following incubation, the sections were treated with peroxidase using the labeled polymer method with EnVision ${ }^{\mathrm{TM}}$ Peroxidase (Dako Corp.) for $60 \mathrm{~min}$. The peroxidase reaction was visualized with the liquid DAB substrate kit (Zymed Laboratories Inc., San Francisco, CA, USA). Sections were counterstained with Mayer's hematoxylin. A consecutive section from each sample processed without the primary antibody was used as a negative control. The positive control consisted of a human breast cancer tissue for MMP-10, tonsil tissue for Ki-67 and kidney tissue for CD34. In situ labeling for apoptosis was performed as described previously (14). We used the Apop Tag In Situ Apoptosis Detection kit (Intergen Company, Purchase, NY, USA), which is based on the terminal deoxynucleotidyl transferase-mediated nick endlabeling (TUNEL) method.

Evaluation. To evaluate immunohistochemical staining for MMP-10, the staining intensity was graded as none, weak, moderate or strong. However, it was difficult to distinguish between weak and moderate staining. Subsequently, carcinoma cells with strong staining intensity were considered as positively stained cells. The proliferation index (PI) was estimated by counting the number of cells with nuclei positively stained for the anti-Ki-67 antibody. The apoptotic index (AI) was estimated by the percentage of TUNEL-positive cells. Semi-quantitative analyses were performed on at least 500 cancer cells in 3-6 different fields per section. From the PI and AI values, we calculated the cell renewal index (CRI=PI/AI). This index was used in previous reports $(3,13)$.

To analyze microvessel density (MVD), tumor sections stained with the anti-CD34 antibody were examined under an E-400 microscope (Nikon, Tokyo, Japan). The digital images were captured using a digital camera (model DU100; Nikon) at x200 magnification. For each tumor section, 3-5 fields with the highest blood vessel density (hot spots) were evaluated. To determine the MVD, defined as the number of vessels per field (x200), we used a computer-aided image analysis system (Win ROOF, version 5.0; Mitani Corp., Fukui, Japan).

To perform logistic regression analyses for the above variables, we divided the tissue samples into two groups: those with values higher than the median value for the entire group, and those with values lower than the group median value. Slides were evaluated twice at different times by two investigators (S.M. and Y.S.) who were blinded to the clinical characteristics and pathological data.

Statistical analysis. Results are expressed as median and interquartile range (IQR) values. The Mann-Whitney U test was performed for continuous variables of the data. The Scheffé test was used for multiple comparisons of the data. Pearson's correlation was used to evaluate the relationship between continuous variables. The correlation coefficient (r) and corresponding P-values were also reported. Spearman's rank correlation coefficient was calculated to confirm Pearson's correlation. Variables that achieved statistical significance 

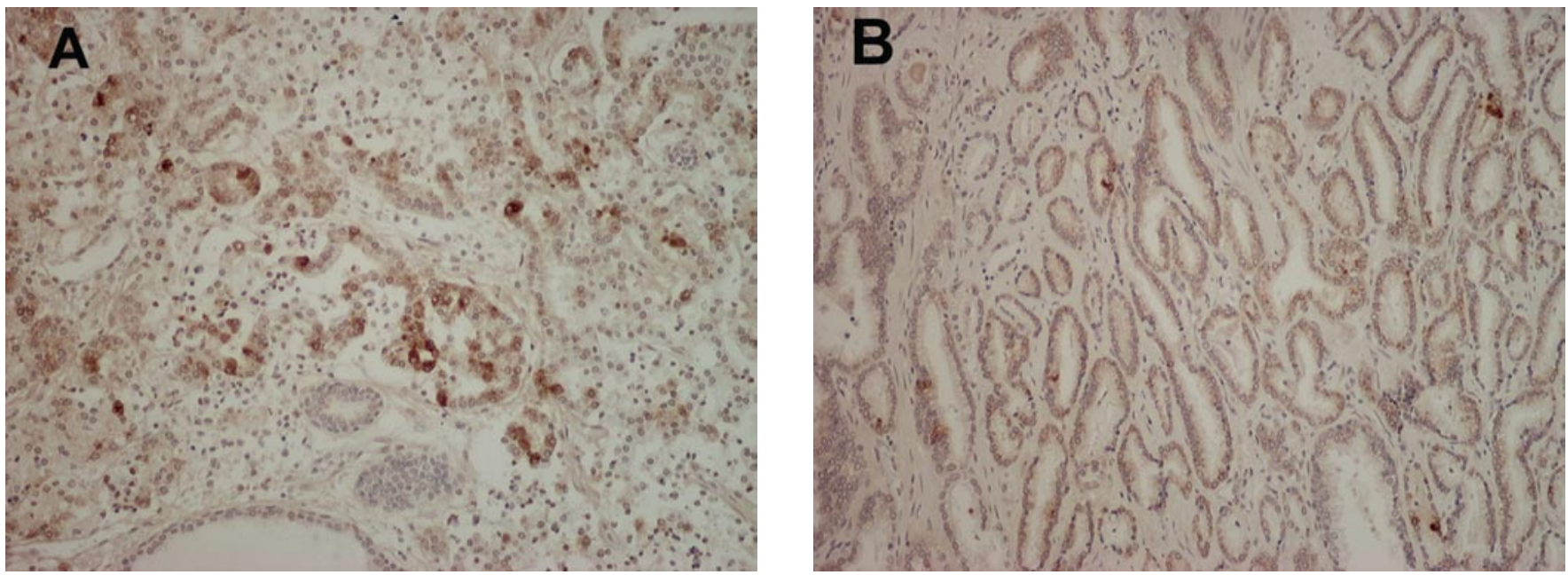

Figure 1. Immunohistochemistry of MMP-10 in prostate tissues of patients with prostate cancer (A) and in non-tumoral glands (B). Immunostaining for MMP-10 was mainly detected in the cytoplasm of cancer cells. Original magnification, x200.

Table I. Relationship between MMP-10 expression and pathological features.

\begin{tabular}{|c|c|c|c|}
\hline & & MMP-10-stained cancer cells & \\
\hline & No. of patients & Median (interquartile range) & P-value \\
\hline \multicolumn{4}{|c|}{ Pathological features } \\
\hline pT stage & & & 0.007 \\
\hline $\mathrm{T} 2$ & 39 & $11.3 \quad(7.6-20.6)$ & \\
\hline $\mathrm{T} 3$ & 24 & $22.3(13.4-30.2)$ & \\
\hline Venous invasion & & & 0.025 \\
\hline Absence & 40 & $11.2 \quad(7.9-22.1)$ & \\
\hline Presence & 23 & $21.8(13.2-26.9)$ & \\
\hline Gleason's score & & & 0.043 \\
\hline Low $(<7)$ & 20 & $10.0(7.1-13.6)$ & \\
\hline Middle (=7) & 21 & $17.9 \quad(9.2-31.2)$ & \\
\hline $\operatorname{High}(>7)$ & 22 & $21.6(11.5-28.0)$ & \\
\hline
\end{tabular}

MMP, matrix metalloproteinase.

$(\mathrm{P}<0.050)$ in the univariate analysis were subsequently entered into a multivariate analysis using logistic regression analysis [described as odds ratios (OR) with $95 \%$ confidence intervals (95\% CI), together with the P-values]. Statistical tests were two-sided, and significance was defined as $\mathrm{P}<0.050$. Statistical analyses were performed on a personal computer with the statistical package StatView for Windows (version 5.0).

\section{Results}

Clinicopathological features. The mean age of patients at surgery was 62 years (range 44-77). Based on a histopathological examination, 39 cases $(61.9 \%)$ in this cohort were diagnosed as pT2 and $24(38.1 \%)$ as pT3. With regard to GS, 2 cases $(3.2 \%)$ had a score of 5,18 cases $(28.6 \%)$ of 6,21 cases $(33.3 \%)$ of 7,9 cases $(14.3 \%)$ of 8 and 13 cases $(20.6 \%)$ had a score of 9 . Thus, the low GS group consisted of 20 patients
(31.7\%), the middle of $21(33.3 \%)$ and the high GS group of 22 patients $(34.9 \%)$.

MMP-10 expression. A representative example of cancer cells positively stained for MMP-10 is shown in Fig. 1A. MMP-10 immunostaining was mainly detected in the cell cytoplasm, and weak staining was noted in some stromal tissues. MMP-10-positive cancer cells were uniformly present throughout the cancer tissue but no unique distribution was noted. On the other hand, in prostate glands that were free of tumors, cells strongly stained for MMP-10 were rare (Fig. 1B). The proportion of MMP-10-stained cancer cells (median $13.8 \%$, IQR $8.8-25.5 \%)$ was significantly higher $(\mathrm{P}<0.001)$ than the non-tumoral gland cells (median $2.4 \%$, IQR $0-3.7 \%$ ).

Correlation with clinicopathological features. As shown in Table I, the proportion of MMP-10-stained cancer cells was 
Table II. The relationship between MMP-10 and parameters.

\begin{tabular}{lrc}
\hline & $\mathrm{r}$ & P-value \\
\hline Proliferation index & 0.230 & 0.068 \\
Apoptotic index & -0.240 & 0.057 \\
Microvessel density & 0.210 & 0.100 \\
Cell renewal index & 0.340 & 0.001
\end{tabular}

significantly higher in pT3 $(\mathrm{P}=0.007)$ than in $\mathrm{pT} 2$ tumors. Furthermore, the proportion of MMP-10-stained cancer cells in high GS tumors was significantly higher $(\mathrm{P}=0.043)$ than in low GS tumors. To test the independent significance of MMP-10 expression for a high pT stage, a multivariate analysis model including MMP-10 and GS was examined. This analysis showed that MMP-10 expression correlated closely and positively with a high pT stage (OR 3.63, 95\% CI 1.14-11.58, $\mathrm{P}=0.029)$. We then investigated the relationship between MMP-10 expression and vascular invasion. As shown in Table I, MMP-10 expression in specimens with vascular invasion was significantly higher $(\mathrm{P}=0.025)$ than those without vascular invasion.

Correlation with proliferation, apoptosis and angiogenesis. We examined the correlation between MMP-10 and PI, AI, MVD and CRI (Table II). Although MMP-10 expression tended to correlate with PI and MVD, the correlations were not statistically significant $(\mathrm{P}=0.068$ and 0.100 , respectively). In contrast, MMP-10 expression correlated negatively with AI, although this correlation showed borderline significance $(\mathrm{P}=0.057)$. On the other hand, MMP-10 expression correlated significantly with CRI (=PI/AI) $(\mathrm{r}=0.34, \mathrm{P}=0.001)$. When the relationship between $\mathrm{pT}$ stage and these parameters was examined, CRI in pT3 (median 5.2, IQR 3.3-8.2) was significantly higher $(\mathrm{P}=0.048)$ than in $\mathrm{pT} 2$ (median 3.9 , IQR 2.2-5.3) tumors. Thus, both MMP-10 and CRI were significantly associated with $\mathrm{pT}$ stage.

\section{Discussion}

The present study found that MMP-10 was overexpressed in prostate cancer cells, and its positively stained ratio in pT3 was significantly higher than in pT2 tumors. In several studies using prostate cancer cell lines, MMP-10 expression levels in cancer cells were significantly higher than those in normal epithelial cells $(16,17)$. Our results, based on the study of human cancer tissues, support the conclusion of the above studies. Therefore, we speculated that MMP-10 is up-regulated during the carcinogenic process, and plays an important role in tumor development in patients with prostate cancer.

Special attention was paid to the pathological roles of MMP-10 at the early stages of tumor progression. To examine the characteristics and distribution patterns of MMP-10 expression in human prostate cancer tissues, we only examined specimens obtained through radical surgery rather than from needle biopsy. In previous reports, a strong expression of certain MMP members was detected at the invasive front of tumors $(18,19)$. However, such unique features were not found in almost all of the specimens in this study. On the other hand, our results demonstrated that MMP-10 was closely associated with pT stage in our multivariate analysis model. Thus, while MMP-10 plays an important role in tumor growth, further detailed examination is necessary regarding its role in prostate cancer. In addition, our results showed that MMP-10 expression was associated with vessel invasion. To the best of our knowledge, this is the first report on such an association. Moreover, this finding confirms that MMP-10 has important pathological significance in the early stages of prostate cancer progression.

To clarify the pathological roles of MMP-10 in human prostate cancer, we investigated the relationship between its expression and PI, AI, MVD and CRI. In other types of malignancies, MMP-10 has been reported to play crucial roles in cancer cell proliferation, apoptosis and angiogenesis $(12,13)$. However, in contrast to our expectation, although some correlation was found between the proportion of MMP-10-stained cells and PI, AI or MVD in prostate cancer, this correlation was not statistically significant. Our results cannot explain such a disparity between the studies. We believe, however, that these differences are due to variations in pathological stage and malignant potential. In particular, our study population did not include patients with local invasion or metastasis. In general, the pathological significance and activity of cell proliferation, apoptosis and angiogenesis in the early stages of tumor development is relatively lower compared to those in advanced cancer types. In addition, it is known that the malignant potential and aggressiveness of prostate cancer, especially in low stage tumors, is relatively lower compared to that of other cancer types. Although PI, AI and MVD in advanced stages of prostate cancer are significantly different from those in the early stages, such differences were not found between pT3 and pT2 tumors $(1,3)$. Therefore, it was difficult to detect a statistically significant correlation between MMP-10 and these parameters in our study population. Thus, we suggest that MMP-10 expression does not correlate significantly with PI, $\mathrm{AI}$ and MVD in patients with organ-confined prostate cancer. On the other hand, MMP-10 expression correlated positively with CRI (=PI/AI). The balance of cancer cell proliferation and apoptosis is one of the strongest determinants of tumor growth. Thus, we presume that CRI reflects cancer cell growth more precisely than PI or AI alone, especially in organ-confined prostate cancer. Several investigators described CRI as a useful marker of true growth activity under physiological and pathological conditions, including malignancy $(15,20)$. Our results showed that there was a significant difference between CRI in pT2 compared to that in pT3 tumors. We speculate that MMP-10 modulates the balance between cell proliferation and the apoptosis of cancer cells in non-metastatic prostate cancer tissues, and this mechanism may play a role in the growth and up-staging of this disease.

In conclusion, our results demonstrated that MMP-10 was up-regulated during the process of carcinogenesis. Moreover, the expression of MMP-10 was significantly and positively associated with $\mathrm{pT}$ stage and GS in patients with non-metastatic prostate cancer. In addition, we speculated that MMP-10 modulates tumor growth and up-staging via the regulation of the balance of cell proliferation and apoptosis. Our results 
provide important findings for planning observation and treatment strategies for patients with non-metastatic prostate cancer.

\section{Acknowledgements}

We are grateful to Mr. Yoshikazu Tsuji and Mr. Takumi Shimogama for the outstanding support. This study was supported by a Grant-in-Aid for Scientific Research from the Ministry of Education, Science, Technology and Culture of Japan (grant no. 17791080).

\section{References}

1. Matsushima H, Goto T, Hosaka Y, Kitamura T and Kawabe K: Correlation between proliferation, apoptosis and angiogenesis in prostate carcinoma and their relation to androgen ablation. Cancer 85: 1822-1827, 1998.

2. Visakorpi T, Kallioniemi O-P, Koivula T and Isola J: New prognostic factors in prostatic carcinoma. Eur Urol 24: 438-449, 1993.

3. Weidner N, Carroll PR, Flax J, Blumenfeld W and Folkman J: Tumor angiogenesis correlates with metastasis in invasive prostate carcinoma. Am J Pathol 143: 401-409, 1993.

4. Egelbald M and Werb Z: New functions for the matrix metalloproteinases in cancer progression. Nat Rev Cancer 2: 161-174, 2002.

5. Ray JM and Stetler-Stevenson WG: The role of matrix metalloproteinases and their inhibitors in tumor invasion, metastasis and angiogenesis. Eur Respir J 7: 2062-2072, 1994.

6. Miyata Y, Iwata T, Ohba K, Kanda S, Nishikido M, Koga S and Kanetake H: Expression of matrix metalloproteinase-7 on cancer cells and tissue endothelial cells in renal cell carcinoma: prognostic implication and clinical significance for invasion and metastasis. Clin Cancer Res 15: 6998-7003, 2006.

7. Wood M, Fudge K, Mohler AR, Frost AR, Garcia F, Wang M and Stearns ME: In situ hybridization studies of metalloproteinase 2 and 9 and TIMP-1 and TIMP-2 expression in human prostate cancer. Clin Exp Metastasis 15: 246-258, 1997.

8. Pavlaki M and Zucker S: Matrix metalloproteinase inhibitors (MMPIs): the beginning of the phase I or the termination of phase III clinical trials. Cancer Metastasis Rev 22: 177-203, 2003.

9. Arlt M, Kopitz C, Pennington KL, Watson KL, Krell HW, Bode W, Gansbacher B, Khokha R, Edward DR and Krüger A: Increase in gelatinase-specificity of matrix metalloproteinase inhibitors correlated with antimetastatic efficacy in a T-cell lymphoma model. Cancer Res 62: 5543-5550, 2002.
10. Miyazaki K, Koshikawa N, Hasegawa S, Momiyama $N$, Nagashima $Y$, Moriyama K, Ichikawa $Y$, Ishikawa $T$, Mitsuhashi M and Shimada H: Matrilysin as target for chemotherapy for colon cancer: use of antisense oligonucleotides as antimetastatic agents. Cancer Chemother Pharmacol 43: S52-S55, 1999.

11. Mathew R, Khanna R, Kumar R, Mathur M, Shukla NK and Ralhan R: Stromelysis-2 overexpression in human esophageal squamous cell carcinoma: potential clinical implications. Cancer Detect Prev 26: 222-228, 2002.

12. Miyata $Y$, Iwata $T$, Maruta $S$, Kanda $S$, Nishikido $M$ and Kanetake H: Expression of matrix metalloproteinase-10 in renal cell carcinoma. Eur Urol 52: 791-797, 2007.

13. Meyer E, Vollmer J-Y, Bovey R and Stamenkovic I: Matrix metalloproteinase 9 and 10 inhibit protein kinase C-potentiated, p-53-mediated apoptosis. Cancer Res 65: 4261-4272, 2005.

14. Miyata Y, Koga S, Kanda S, Nishikido M, Hayashi T and Kanetake H: Expression of cyclooxygenase-2 in renal cell carcinoma: correlation with tumor cell proliferation, apoptosis, angiogenesis, expression of matrix metalloproteinase-2 and survival. Clin Cancer Res 9: 1741-1749, 2003.

15. Bai M, Agnantis N, Kamina S, Demou A, Zagorianakou $P$, Katsaraki A and Kanavaros P: In vivo cell kinetics in breast carcinogenesis. Breast Cancer Res 3: 276-283, 2001.

16. Singh S, Singh UP, Grizzle WE and Lillard JW Jr: CXCL12CXCR4 interactions modulate prostate cancer cell migration, metalloproteinase expression and invasion. Lab Invest 84: 1666-1676, 2004

17. Singh S, Singh UP, Stiles JK and Lillard JW Jr: Expression and functional role of CCR9 in prostate cancer cell migration and invasion. Clin Cancer Res 10: 8743-8750, 2004.

18. Adachi Y, Yamamoto H, Itoh F, Arimura Y, Nishi M, Endo T and Imai K: Clinicopathologic and prognostic significance of matrilysin expression at the invasive front in human colorectal cancers. Int J Cancer 95: 290-294, 2001.

19. Gu ZD, Li JY, Li M, Gu J, Shi XT, Ke Y and Chen KY: Matrix metalloproteinase expression correlates with survival in patients with esophageal squamous cell carcinoma. Am J Gastroenterol 100: 1835-1843, 2005

20. Navarrete MA, MaierCM,Falzoni R, Gerk de Azevedo Quadros L, Kima GR, Baracat EC and Nazário AC: Assessment of the proliferative, apoptotic and cellular renovation indices of the human mammary epithelium during the follicular and luteal phases of the menstrual cycle. Breast Cancer Res 7: R306-R313, 2005. 